\title{
MODELS OF ROTATING WOLF-RAYET STARS
}

\author{
J. FLIEGNER and N. LANGER \\ MPI für Astrophysik, D-85740 Garching, B.R. Deutschland
}

\begin{abstract}
We explore the influence of rotation on the evolution of massive stars $\left(40 \mathrm{M}_{\odot}\right)$. We find the evolution in the HRD, the initial-final mass relation, and the duration of the various WR phases to depend sensitively on the initial rotation rate. We predict that, independent of the initial rotation rate, the majority of WR stars should be slow rotators.
\end{abstract}

Among high mass main sequence (MS) stars moderate to fast rotation is observed as a common feature. Their evolution may not only be influenced by a reddening due to the reduced effective gravity, but even more by rotationally induced mixing processes (e.g., Endal \& Sofia 1978). As a consequence, a considerably altered internal hydrogen profile is expected, which may strongly affect the evolution through the Wolf-Rayet stages, especially for fast rotating stars.

Here, we discuss the effects of rotation for a $40 \mathrm{M}_{\odot}$ star. Three sequences of stellar models with different initial rotational velocities are calculated (sequences R1, R2, R3, cf. Tab. 1), and compared with the evolution of a non-rotating model (sequence S1). The effects of rotation on the stellar structure as well as rotationally induced mixing are computed following Pinsonneault et al. (1989).

With increasing rotational velocity and therefore faster mixing, the models show higher luminosities and temperatures during their MS evolution (cf. Fig. 1), and an increased MS life-time (cf. Tab. 1). For slower rotation the induced mixing affects only weakly the stellar surface conditions; the surface helium abundance is increased by $2 \%$ (R1) and $11 \%$ (R2). However, for fast rotation the whole star is mixed, though not completely homogenized ( $c f$. Figs. 3 and 4). The surface hydrogen abundance drops below $40 \%$ during central $\mathrm{H}$ burning. Consequently, WR mass loss is adopted in this case. The rapid WR mass loss leads to a strong decrease in luminosity ( $c f$. Maeder 1987) and rotational velocity (cf. Figs. 1 and 2; cf. Maheswaran \& Cassinelli 1994). At core hydrogen exhaustion, a helium star of $5.3 \mathrm{M}_{\odot}$ with a surface velocity of only $10 \mathrm{~km} \mathrm{~s}^{-1}$ is left. Because of the low velocity and the short time-scale of helium burning, nearly no rotationally induced mixing is performed during core helium burning. In particular, no products of He burning are mixed to the surface (cf. Fig. 5). Note, that for about $15 \%$ of the WR life-time the surface velocity is above $50 \mathrm{~km} \mathrm{~s}^{-1}$ (cf. Fig. 2), which may be relevant for polarimetric observations.

Fig. 3 compares hydrogen abundances and luminosities of the sequences R3 and S1 with observed galactic OB (asterisks),WNL (triangles), WNE-s (diamonds) and WNE-w stars (squares) (cf. Langer et al. 1994). The dark 


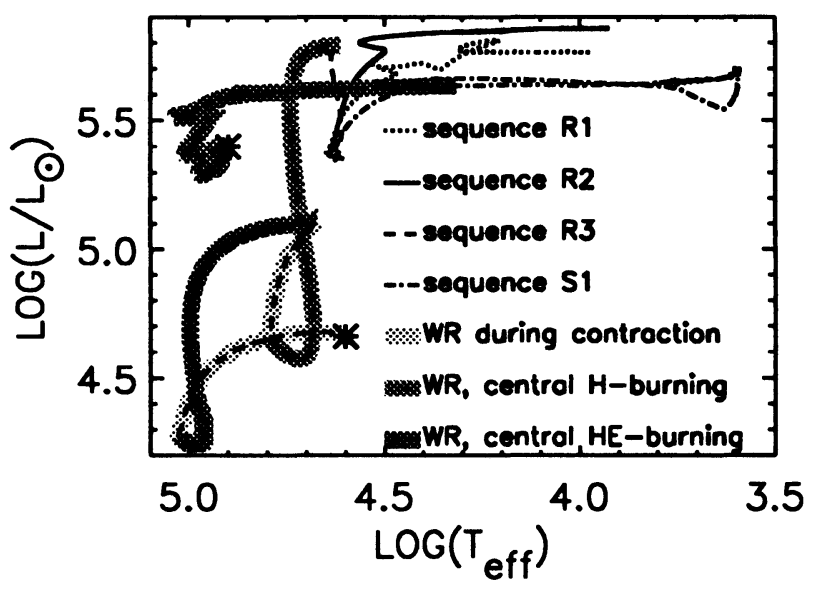

Fig. 1. The evolution of our models in the HR-diagram. WR phases are shaded.
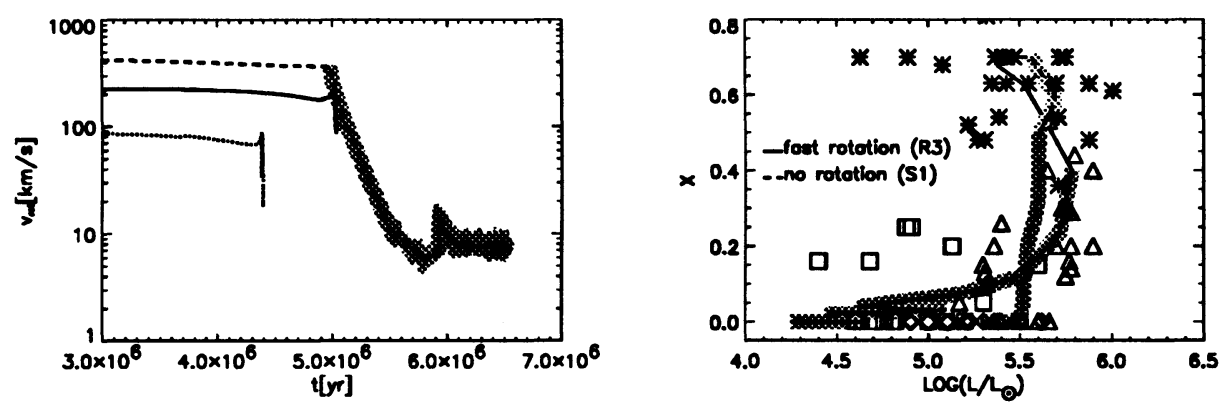

Fig. 2. The evolution of the surface velocity Fig. 3. Surface hydrogen abundance versus of our models. Lines are coded as in Fig. 1. luminosity for the sequences S1 and R3.

shaded part of the theoretical track marks the WR phase of the models R3 and $\mathrm{S} 1$, the light shaded part corresponds to the red supergiant stage of model S1 (cf. Fig. 1).

In summary, though moderate rotation may have only limited effect, rapid rotation can completely modify the evolution of a massive star. The MS life-time and the evolutionary track in the HR-diagram are changed. The WR phase, which the standard picture predicts to occur during late core helium burning after a red supergiant phase, is reached during central hydrogen burning (cf. Fig. 1). As a consequence, the duration of the WR phase and the duration of the hydrogen free evolution are altered (cf. Tab. 1). Due to the strong WR mass loss, the star (R3) looses most of its angular momentum and undergoes central He burning as a slow rotator, in contrast 


\section{TABLE I}

Surface velocity at the ZAMS, MS life-time, duration of core He burning, duration of the WR phase compared to the MS life-time and duration of the hydrogen free evolution compared to the WR life-time of our models.

\begin{tabular}{rrrrrr}
\hline model & $v_{\text {rot }}(\mathrm{km} / \mathrm{s})$ & $t_{H}(\mathrm{Myr})$ & $t_{H e}(\mathrm{Myr})$ & $t_{W R} / t_{H}$ & $t_{H-f r e e} / t_{W R}$ \\
\hline S1 & 0 & 4.1 & 0.31 & 0.083 & 0.754 \\
R1 & 100 & 4.3 & presently & not & available \\
R2 & 260 & 5.0 & presently & not & available \\
R3 & 430 & 5.9 & 0.95 & 0.396 & 0.488 \\
\hline
\end{tabular}

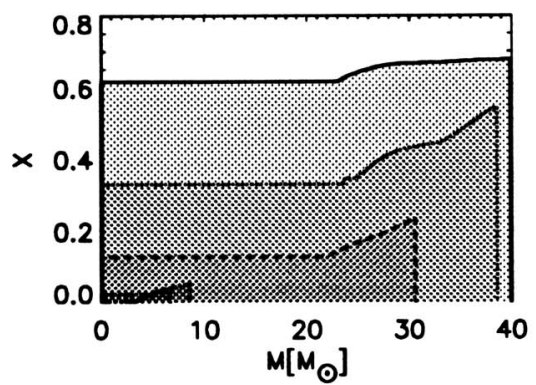

Fig. 4. The evolution of the hydrogen pro- Fig. 5. The evolution of the helium profile file of sequence R3 during central H-burning. of sequence R3 during central He-burning.

to our initially slowly rotating models (cf. Fig. 2). The evolutionary track of our initially rapidly rotating star in the HR diagram (R3, cf. Fig. 1) can account for some otherwise unexplained features. Most important, it predicts hydrogen containing WR stars of relatively low luminosity $(4.6 \leq$ $\left.\log L / \mathrm{L}_{\odot} \leq 5.1\right)$ and also faint hydrogen-free WN stars (cf. Figs. 1 and 5). The determination of rotational velocities for the intrinsically faintest WN stars would provide a most interesting test for our calculations.

Acknowledgement This work has been supported by the Deutsche Forschungsgemeinschaft through grant La 587/8-1

\section{References}

Endal, A.S., Sofia, S. 1978, $A p J$ 220, 279

Langer, N., Hamann, W.-R., Lennon, M., Najarro, F., Pauldrach, A.W.A., Puls, J. 1994, $A \mathcal{E} A$, in press

Maeder, A. 1987, $A \& A$ 178, 159

Maheswaran, M., Cassinelli, J.P. 1994, in press

Pinsonneault, M.H., Kawaler, S.D., Sofia, S., Demarque, D. 1989, ApJ 338, 424 